\title{
La cirugía laparoscópica por endometriosis profunda es efectiva en el manejo del dolor
}

\author{
Guillermo Durruty V. ${ }^{1}$, Claudia Celle $T^{1}{ }^{1}$, Milena Zamboni $T^{1}{ }^{1}$, Cristian Pomés C. ${ }^{1}$ \\ ${ }^{1}$ Unidad de Ginecología, División de Obstetricia y Ginecología, Facultad de Medicina, Pontificia Universidad Católica de \\ Chile.
}

\section{RESUMEN}

Antecedentes: La endometriosis profunda (EP) es una causa frecuente de algia pélvica crónica que afecta negativamente la calidad de vida de las pacientes. La cirugía, idealmente por vía laparoscópica, es el tratamiento de elección para pacientes sintomáticas. Objetivo: Evaluar la cirugía laparoscópica en el control del dolor en la EP. Método: Mediante un estudio retrospectivo se analizaron a todas las pacientes operadas por endometriosis profunda con el objetivo de evaluar el alivio sintomático al corto y largo plazo. Resultados: El $85 \%$ y $83 \%$ de las pacientes refirió alivio sintomático al año y dos años de seguimiento respectivamente. En pacientes con endometriosis del tabique rectovaginal y extenso compromiso rectosigmoideo este porcentaje alcanza un $89 \%$ que se mantiene a los 2 años. Conclusión: La cirugía laparoscópica es el tratamiento de elección de la EP cuando es sintomática, logrando alivio sintomático significativo que es persistente en el tiempo. En manos de cirujanos expertos la cirugía para EP es segura y logra resultados quirúrgicos satisfactorios en términos de remisión sintomática con baja tasa de complicaciones mayores.

\section{PALABRAS CLAVE: Endometriosis profunda, algia pélvica crónica, endometriosis tabique rectovaginal}

\section{SUMMARY}

Background: Deep infiltrating endometriosis (DIE) is a common cause of chronic pelvic pain which negatively affects the quality of life of patients. Laparoscopic surgery is the treatment of choice when patients are symptomatic. Objective: To evaluate laparoscopic surgery in the control of pain for DIE. Methods: We retrospectively studied all patients who underwent surgery for DIE in order to evaluate short and long term symptom relief. Results: Patients at 1 and 2 year follow-up referred $85 \%$ and $83 \%$ of symptomatic relief respectively. In patients with DIE specifically compromising the rectosigmoid colon this relief reached $89 \%$ and was stable after 2 year follow-up. Conclusion: Laparoscopic surgery is the treatment of choice for symptomatic DIE achieving significant symptomatic relief that is persistent over time. In ther hands of expert surgeons surgery for DIE is safe and it achieves satisfactory surgical results in terms of symptomatic remission with low mayor complication rates.

KEY WORDS: Deep infiltrating endometriosis, chronic pelvic pain, rectovaginal endometriosis 


\section{INTRODUCCIÓN}

La endometriosis es una causa frecuente de dolor pélvico crónico en mujeres de edad fértil. Es una enfermedad que afecta negativamente la calidad de vida y es responsable de un porcentaje no despreciable de ausencias escolares y laborales. Independiente de la magnitud o grado de la enfermedad el dolor es el síntoma principal (1).

La endometriosis se subdivide en 3 tipos ampliamente conocidos, la endometriosis peritoneal, la endometriosis quística del ovario y la endometriosis del tabique rectovaginal (2). En los 3 tipos existe dolor referido como algia pélvica crónica, dismenorrea, disquexia y dispareunia profunda. Estos últimos dos particularmente en relación a endometriosis del tabique rectovaginal, donde además suele existir dolor a la palpación de los ligamentos uterosacros, presencia de nodularidad y/o retracción de los ligamentos, e incluso la palpación de un tumor duro retrocervical.

La EP genera inflamación y retracción de tejidos vecinos, y en este proceso puede comprometer órganos ginecológicos, recto y vejiga. La presencia de disquexia, diarrea y/o rectorragia catamenial deben hacer pensar en endometriosis con compromiso de recto o colon rectosigmoides. Así mismo disuria o poliaquiuria catamenial deben levantar la sospecha de compromiso vesical y buscarlos dirigidamente dentro del estudio de la enfermedad. En más del $95 \%$ de los casos la EP se asocia a nódulo palpable y dolor severo. En un $5-12 \%$ de pacientes la EP compromete el colon rectosigmoides (3).

La cirugía de la endometriosis profunda es difícil, con un alto riesgo de complicaciones mayores producto de la inflamación, vascularización, distorsión anatómica y secundariamente alteración de los planos quirúrgicos $(1,4)$. Dado el alto riesgo de daño de órganos como uréter y colon esta intervención debe estar reservada para cirujanos altamente entrenados. Esta cirugía puede ser realizada por una laparotomía tradicional, pero las limitantes en la visión de los planos quirúrgicos la hace de muy alto riesgo, por lo que la cirugía por vía laparoscópica es la vía de abordaje de elección en el tratamiento de la endometriosis profunda.

Si bien el tratamiento médico es la primera línea en el manejo del dolor por endometriosis, este suele ser muchas veces insuficiente. El tratamiento de elección para los casos que no responden a tratamiento médico es la cirugía, idealmente por vía laparoscópica. Los objetivos quirúrgicos son resecar la mayor cantidad de enfermedad para lograr el alivio sintomático, sin olvidar la importancia de respetar la funcionalidad y anatomía de los órganos pélvicos durante el acto quirúrgico (5), más aun si consideramos que esta enfermedad afecta a mujeres en edad reproductiva.
Es bien conocido que la menstruación puede acompañarse de algún grado de dolor, pero en ningún caso este debiera ser invalidante por lo que ante esta situación el médico debiera proceder investigar y descartar la presencia de endometriosis profunda. Nuestra institución es centro de referencia de esta patología y como tal el número de cirugías ha ido aumentando progresivamente en los últimos años y en especial la cirugía por endometriosis profunda.

El objetivo principal de este trabajo es evaluar el alivio sintomático en las pacientes operada por esta patología en nuestro centro. El objetivo secundario es evaluar la seguridad y eficacia de la cirugía conservadora para el tratamiento de la EP.

\section{PACIENTES Y MÉTODOS}

Se efectuó un estudio retrospectivo de todas las pacientes operadas de endometriosis pélvica en el Hospital Clínico de la Pontificia Universidad Católica entre los años 2004 y 2013. Se incluyeron todos los casos con diagnóstico o sospecha de endometriosis que fueron a cirugía. De éstas se analizaron los casos correspondientes a EP que incluyó a pacientes con endometriosis del tabique rectovaginal (ETRV) y endometriosis de ligamentos uterosacros (EUS). No se excluyeron pacientes.

Para la recopilación de datos se utilizaron registros clínicos, base de datos de protocolos operatorios y esquemas quirúrgicos. Se realizó además contacto telefónico a aquellas pacientes cuyo seguimiento fuera insuficiente o incompleto.

Se realizó un análisis descriptivo de características etarias, paridad, cirugías previas de la muestra estudiada, distribución de las distintas formas de endometriosis, tiempo operatorio, complicaciones mayores intra y postoperatorias, estadía hospitalaria, tasa de embarazo y remisión sintomática.

Se consideraron como complicaciones mayores las lesiones urológicas (vesicales y ureterales), lesiones rectales, complicaciones hemorrágicas e infecciosas. No se consideraron como complicación los casos de deserosamiento rectal pues dada la dificultad de la cirugía se considera un evento esperable.

Técnica quirúrgica: En todas las pacientes se realizó una resección dirigida de las lesiones endometriósicas, principalmente nodulares siguiendo las normas de seguridad bajo las cuales establecimos nuestra técnica quirúrgica estandarizada (TQE). La TQE consistió en disección roma y cuidadosa de los espacios avasculares de la pelvis, identificación y restablecimiento de reparos anatómicos (aislamiento y lateralización del uréter y desarrollo de espacios pararectales), utilización de energía bipolar y resección en pantalón del nódulo o úterosacro afectado con o sin resección o apertura de vagina y/o recto comprometido. 


\section{RESULTADOS}

En el período de estudio se operaron 785 casos de endometriosis pélvica. De ellas, un $26 \%(n=204)$ correspondieron a EP $(79 \%$ cirugías por ETRV y $21 \%$ por EUS). El $84 \%(n=171)$ de las biopsias confirmaron endometriosis. El promedio de edad fue de 33 años (Mediana 33 años, rango: 19-45 años). Del total de 204 pacientes con diagnóstico de EP el $77 \%$ eran nuligestas y un $43 \%$ de ellas tenía diagnóstico de infertilidad además del dolor.

El dolor manifestado como dismenorrea, dispareunia profunda o disquexia fue la principal indicación de cirugía en el 100\% de las pacientes (Figura 1). El $99 \%$ de los casos se abordó por vía laparoscópica. El tamaño del nódulo resecado osciló entre $1 \mathrm{a} 5 \mathrm{~cm}$.

En $12,7 \% \quad(n=26)$ de las cirugías fue necesaria la apertura y/o resección vaginal realizándose cierre de la misma con puntos intracorpóreos por vía laparoscópica sin incidentes. En 1,5\% $(n=3)$ fue necesaria la apertura o resección parcial del rectosigmoides. El tamaño del nódulo fue de 3 y $6 \mathrm{~cm}$ en 2 de estos casos.

El tiempo operatorio promedio fue de 175 minutos (Mediana $170 \mathrm{~min}$, rango 50-575 min). Hubo un $1,5 \%(n=3)$ de conversión a vía abierta dada la gran extensión de la enfermedad. La primera paciente presentaba un nódulo de $6 \mathrm{~cm}$ con compromiso rectal y ureteral, en ella se realizó la cirugía por vía laparoscópica incluida una resección anterior baja (RAB), la conversión fue realizada por el equipo de urología para realizar el reimplante ureteral. En los otros 2 casos la conversión a vía abierta se debió a dificultad técnica por la magnitud y extensión de la enfermedad.

Complicaciones mayores intra y postoperatorias: En nuestra serie hubo un $4 \%(n=8)$ de complicaciones mayores. Dos fueron intraoperatorias y 6 postoperatorias. De las complicaciones intraoperatorias una rexis vesical y una lesión de recto. Ambas corregidas durante el mismo acto quirúrgico con evolución favorable posterior. De las complicaciones postoperatorias $(n=6)$ hubo una inmediata correspondiente a un tromboembolismo pulmonar (TEP). Las otras 5 fueron tardías. De éstas, 4 fueron de origen infeccioso: un absceso tubo-ovárico, un absceso de cúpula vaginal, una fístula tubovaginal secundaria a una celulitis de cúpula, que requirió corrección del defecto a los 5 meses y un plastrón retrouterino que requirió de salpingectomía a los 4 meses por hidrosalpinx. $Y$ la última, 1 caso $(0,5 \%)$ de perforación rectal inadvertida en el intraoperatorio, que se presentó a los 7 días de postoperada

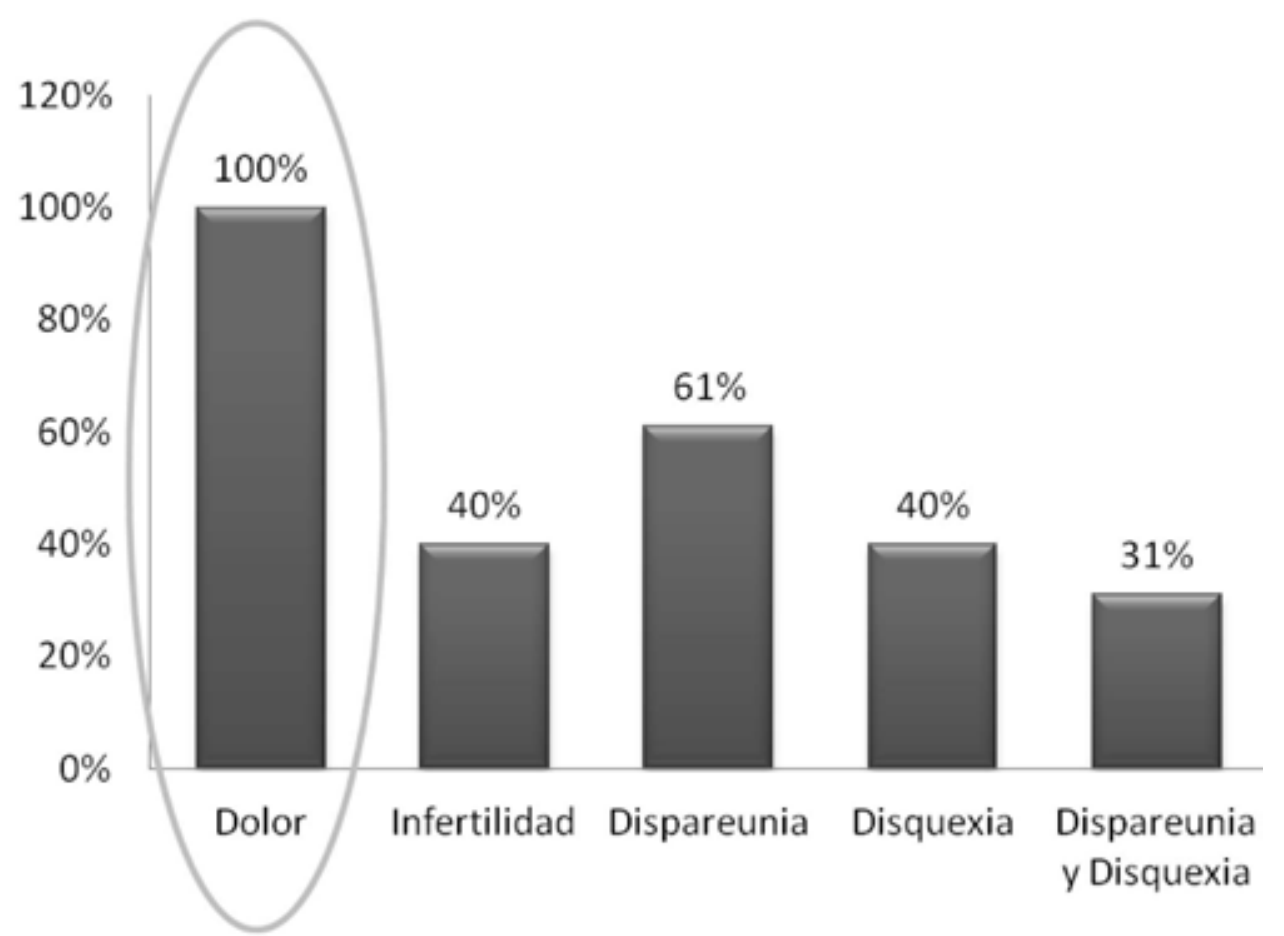

Figura 1. Indicación quirúrgica en endometriosis profunda. 
con un cuadro de abdomen agudo secundario a peritonitis que fue reintervenida de urgencia y requirió una colostomía de Hartmann, corregida en segundo tiempo quirúrgico.

Pese a que el $40 \%$ de las pacientes habían sido sometidas al menos a una cirugía por endometriosis previamente, del total de complicaciones del intraoperatorio, solo una ocurrió en pacientes con el antecedente y correspondió a la lesión vesical ya descrita que se identificó y reparó en el mismo acto quirúrgico.

Post operatorio y alivio sintomático: El tiempo de estadía del postoperatorio fue en promedio de 2,9 días (Mediana 3 días: rango 2-6 días). El 90\% de las pacientes refirió mejoría sintomática significativa en el control de los 3 meses. A 1 y 2 años de seguimiento de la cirugía, un $85 \%$ y $83 \%$ respectivamente lograban el alivio sintomático. En el grupo de pacientes con extenso compromiso rectosigmoideo, un $89 \%$ presentaba un claro alivio sintomático al cabo de 1 año, cifra que se mantuvo estable en el seguimiento de este último grupo de pacientes completados los 2 años.

\section{DISCUSIÓN}

La EP es una patología cada vez más prevalente y nuestro hospital se ha transformado en un centro de referencia nacional llegando a operar más de 50 casos al año en los últimos 2 años (Figura 2). Nuestra técnica es principalmente conservadora (sin resección digestiva) que en la mayoría de los casos logra tasas de alivio global al año de $85 \%$, cifra que disminuye a un $83 \%$ al cabo de 2 años. En pacientes con extenso compromiso rectosigmoideo este porcentaje alcanza un $89 \%$ que se mantiene a los 2 años, demostrando que el alivio logrado en el postoperatorio inmediato suele ser persistente en el tiempo. El éxito de la cirugía rectovaginal por endometriosis ya ha sido ampliamente validado con cifras que superan el $85 \%$ de alivio sintomático en algunas series, lo que se correlaciona con los hallazgos de nuestro estudio $(1,4)$.

La EP puede alterar severamente la anatomía de los órganos pélvicos aumentando considerablemente el riesgo quirúrgico. Es por esto que las complicaciones más frecuentemente descritas son las lesiones de vías urinarias (principalmente ureterales) y las perforaciones digestivas. Dentro de las complicaciones en el postoperatorio las más temidas son las que ocurren tardíamente secundarias a lesiones inadvertidas de las vías urinarias y sobretodo del tracto digestivo. Esta última es una complicación que suele ser grave. Se estima en la literatura una tasa de complicaciones de $3,4 \%$ en cirugía por EP que puede alcanzar hasta un $20 \%$ cuando existe resección colorectal concomitante (6). La serie de Donnez y cols (3), reporta una incidencia de $1,4 \%$ de perforaciones rectales y $0,8 \%$ de lesiones ureterales específicamente. En nuestra serie se reportó sólo un caso de lesión digestiva en más de 200 pacientes y ningún caso de lesión ureteral.

La incidencia de endometriosis ureteral está dada principalmente por reportes de casos y se describe su presencia en aproximadamente un $6 \%$ de las pacientes. La ausencia de esta complicación en nuestra serie se debe a que en nuestra técnica uno de los objetivos principales previo al abordaje del nódulo es identificar, liberar y lateralizar el

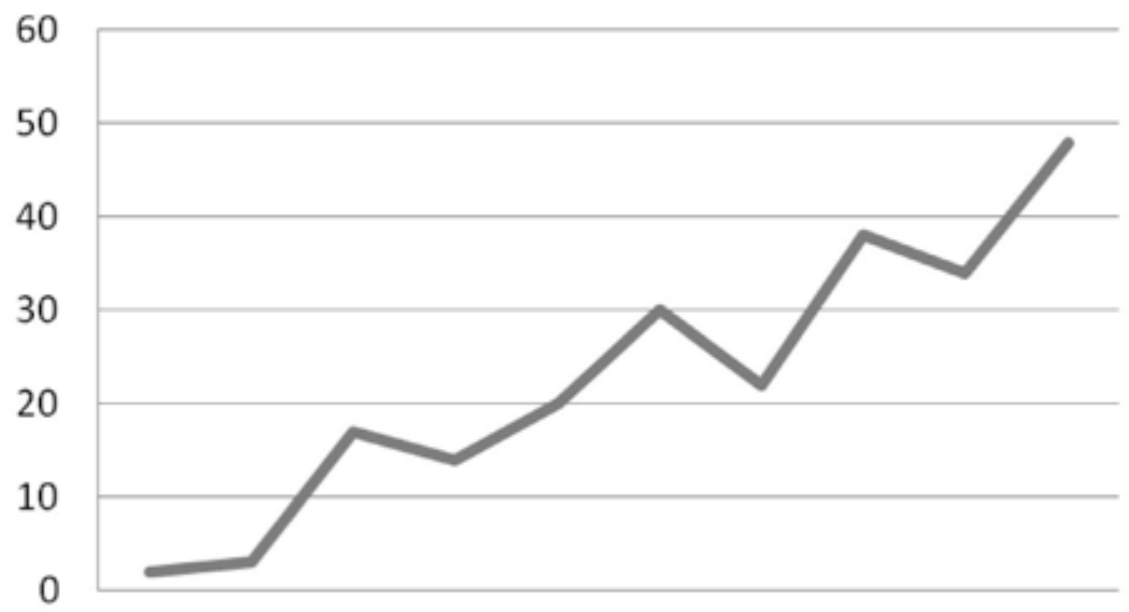

2004200520062007200820092010201120122013

Figura 2. Número de cirugías por endometriosis por año. 
uréter evitando denudarlo y realizando hemostasia precisa y controlada. Disminuyendo de esta forma también lesiones inadvertidas ureterales y sus respectivas complicaciones a corto y largo plazo. En nuestra serie tampoco hubo casos de fistulas rectovaginales, complicación tardía que afecta severamente la calidad de vida de las pacientes. Esta situación se explica por la baja incidencia de apertura simultánea de vagina y recto durante nuestras intervenciones.

La complicación infecciosa es poco frecuente en cirugía por EP. En nuestra serie hubo sólo 3 casos de infección de la cúpula vaginal. Cifra que nos parece alta en relación al número de resecciones con apertura vaginal. No tenemos una explicación evidente a esto, nuestra técnica de aseptización preoperatoria y de aseo intraoperatorio es sistemática. A todas se les realiza un aseo vaginal, el cierre de la cúpula es intracorpóreo con sutura tipo Vicryl, la hemostasia es dirigida pero no excesiva y además utilizamos antibióticos de forma profiláctica en estos casos. Dentro de nuestra hipótesis es que puede deberse al ambiente inflamatorio propio de la endometriosis, al daño vascular y necrosis de tejido secundario a la hemostasia. En ninguno de estos 3 casos hubo apertura simultanea de vagina y de recto, lo que se sabe aumenta la frecuencia de fistulas rectovaginales y podría explicar además una mayor ocurrencia de procesos inflamatorios e infecciosos (6).

Nuestra estrategia de no resecar colon en forma primaria nos llevó a realizar RAB en solo 3 pacientes, todas en el primer año de la serie, pese a que estamos conscientes de que aun en los grupos más agresivos esta cifra nunca alcanza el $100 \%$, y que incluso nunca debiera sobrepasar el 50\% según recomendación de expertos (1). En estos 3 casos fueron resecciones anteriores donde la intervención estaba definida como plan estratégico inicial. Todas se llevaron a cabo con cierre primario.

Nuestra aproximación conservadora a la resección intestinal, aún en los casos de compromiso severo del recto, se ha visto avalada por una muy buena remisión de la sintomatología digestiva pese a haber dejado en algunos casos importante cantidad de tejido fibrótico sobre la cara anterior del recto. Dentro del periodo de estudio ninguna de nuestras pacientes requerió reintervención quirúrgica por reaparición o persistencia de sintomatología digestiva. Lo que refuerza la teoría de que el tejido remanente corresponde con una alta probabilidad a fibrosis y tejido endometriósico cicatricial inactivo (1). En aquellas pacientes que tenían el rectosigmoides comprometido, en menor o mayor medida, el alivio sintomático alcanzó el $89 \%$ a los 2 años de seguimiento.

El abordaje quirúrgico de la endometriosis profunda es peligroso y requiere de una cirugía laboriosa. Por este motivo la indicación quirúrgica debe ser balanceada contra los riesgos. En nuestro grupo el $100 \%$ de las pacientes fue operada por dolor, la mayoría de las veces invalidante. Nuestra vía de abordaje es casi $100 \%$ laparoscópica y los únicos 2 casos de laparotomía primaria fueron dentro de los primeros 10 casos reclutados de la serie dentro del primer año de estudio, lo que apoya firmemente nuestra curva de aprendizaje.

La cirugía laparoscópica es el tratamiento de elección de la EP cuando es sintomática. Las complicaciones más temidas son urológicas e intestinales. A la fecha en nuestro centro se realizan entre 40 a 50 cirugías por EP al año, todas por vía laparoscópica con una tasa de complicaciones urológicas e intestinales de un 1,5\% apoyando nuestra experiencia en esta materia. Nosotros no realizamos resección intestinal de rutina. Siendo el objetivo principal de la cirugía la mejoría del dolor, nuestros resultados muestran que una cirugía agresiva pero prudente y conservadora, es segura y permite obtener resultados quirúrgicos satisfactorios en términos de remisión sintomática con baja tasa de complicaciones mayores.

\section{CONCLUSIÓN}

La cirugía laparoscópica es el tratamiento de elección de la EP cuando es sintomática, logrando alivio sintomático significativo que es persistente en el tiempo. En manos de cirujanos expertos la cirugía para EP es segura y logra resultados quirúrgicos satisfactorios en términos de remisión sintomática con baja tasa de complicaciones mayores.

\section{REFERENCIAS}

1. Koninckx PR, Ussia A, Adamyan L, Wattiez A, Donnez J. Deep endometriosis: definition, diagnosis and treatment. Fertil Steril 2012;98:564-71.

2. Donnez J, Squifflet J. Complications, pregnancy and recurrence in a prospective series of 500 patients operated on by the shaving technique for deep rectovaginal endometriotic nodules. Hum Reprod 2010;25:1949-58.

3. Cicco C, Corona R, Schonman R, Mallova K, Ussia A, Koninckx PR. Bowel resection for deep endometriosis: a systematic review. BJOG 2011;118:285-91.

4. Dunselman G, Vermeulen N, Becker C, Calhaz-Jorge C, D'Hooghe T, De Bie B, Heijinheimo O, Horne A, Keisel L, Nap A, Prentice A, Saridogan E, Soriano D, Nelen W. ESHRE Guideline: management of women with endometriosis. Hum Reprod 2014;29(3):400-12.

5. Johnson N, Hummelshoj L. Consensus on management of endometriosis. Hum Reprod 2013;28(6):155268.

6. Kondo W, Bourdel N, Tamburro S, Cavoli D, Jardon K, Rabischong B, Botchorishvili R, Pouly JL, Mage G, Canis M. Complications after surgery for deeply infiltrating pelvic endometriosis. BJOG 2011;118:292-8. 